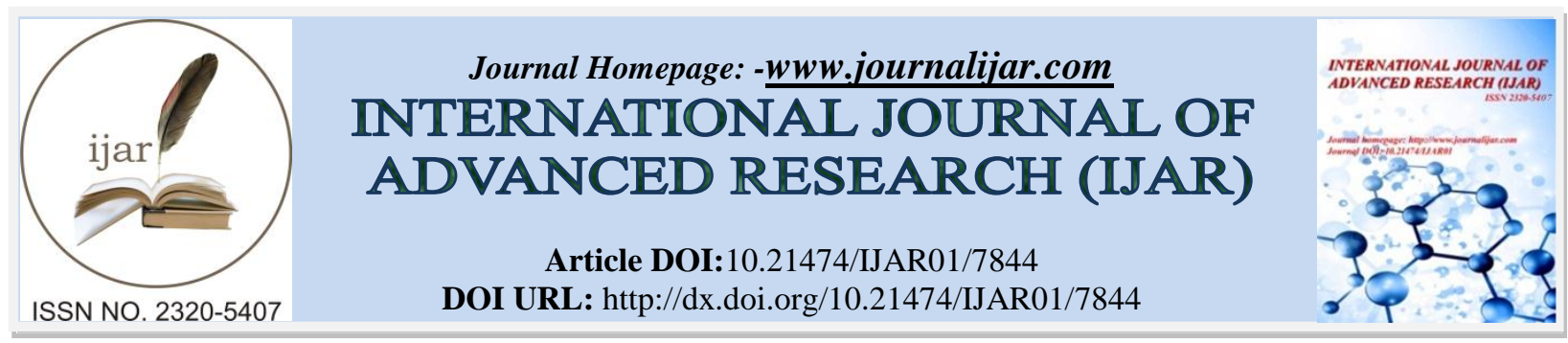

RESEARCH ARTICLE

\title{
STABILITY STUDY OF POLYPHENOLS EXTRACTED FROM THE LEAF SHEATH OF RED SORGHUM (SORGHUM CAUDATUM) FROM BENIN.
}

\section{Agbangnan Dossa cokou pascal ${ }^{1 *}$, Gbohaïda virginie ${ }^{1},{\text { Koudoro yaya } \text { alain }^{1}, \text { Kanfon }^{1} \text { rose estelle }}^{1}$, Wotto Dieudonne valentin ${ }^{2}$ and Sohounhloue C. K. Dominique ${ }^{1}$.}

1. Laboratoire d'Etude et de Recherche en Chimie Appliquée (LERCA), Ecole Polytechnique d'Abomey-Calavi (EPAC), Université d'Abomey-Calavi (UAC), 01 BP 2009 Cotonou République du Bénin.

2. Laboratoire de Chimie Physique (LCP), Faculté des Sciences et Techniques (FAST), Université d'AbomeyCalavi (UAC), République du Bénin.

\section{Manuscript Info}

\section{Manuscript History}

Received: 8 August 2018

Final Accepted: 10 September 2018

Published: October 2018

Keywords:-

Température, polyphénols, sorgho rouge, conservation, $\mathrm{pH}$, decomposition.

\section{Abstract}

L'extrait brut aqueux de la gaine foliaire du sorgho rouge est très utilisé au Bénin et dans la sous-région ouest-africaine à des fins alimentaires et/ou pharmacologiques. Cependant, les conditions de son extractionconservation (forte température, $\mathrm{pH}$ basique, milieu aqueux) laissent croire à une éventuelle dégradation des molécules pouvant réduire l'activité attendue des extraits. La présente étude a pour but d'évaluer l'influence des conditions d'extraction-conservation sur la stabilité des extraits. A cet effet, l'extrait brut réalisé à partir de la poudre de la gaine foliaire du sorgho a été soumis à différentes conditions de $\mathrm{pH}$ (acide, neutre et basique), de température (basse température : $4^{\circ} \mathrm{C}$ et température ambiante) et de luminosité (à la lumière du jour et à l'abri de la lumière) pendant un mois. Un prélèvement de chaque échantillon est réalisé tous les sept jours, pour l'analyse des différentes familles des composés phénoliques par spectrophotométrie. Des résultats obtenus il ressort qu'à $\mathrm{pH}$ neutre, les anthocyanes se conservent mieux à froid (moins de 5\% de dégradation) qu'à température ambiante (18 à $20 \%$ de dégradation). Mais à température égale, la lumière n'a pratiquement pas d'effet sur les pigments anthocyaniques du sorgho (moins de $2 \%$ de dégradation à la lumière après 28 jours). Toute famille confondue, le milieu neutre ou légèrement acide à basse température et à l'obscurité constitue les conditions optimales pour la conservation de l'extrait aqueux du sorgho rouge.

Copy Right, IJAR, 2018,. All rights reserved.

\section{Introduction:-}

De nombreuses études épidémiologiques ont démontré les effets nutritionnels bénéfiques d'unrégime riche en fruits et légumes. Consommés en quantités importantes, ils seraient associés à une réduction des risques de maladies cardio-vasculaires et de certains cancers (Cécile, 2010). Ces bienfaits sont attribués à une teneur élevée en antioxydants, essentiellement les composés phénoliques. Vue la structure chimique de ces composés, plusieurs facteurs extérieurs (température, $\mathrm{pH}$, lumière...) sont susceptibles d'induire des modifications profondes de leur structure et par conséquent de leur activité (Pascal et al. 2011). Néanmoins, les données de la littérature concernant l'évolution des composés phénoliques dans la matrice végétale au cours de sa transformation sont rares. Le plus 
souvent, seules les teneurs en composés phénoliques totaux ou en flavonoïdes totaux ont été évaluées (Singleton, 1999 ; Kabran et al., 2011 ; Fahima et al., 2018). Ainsi, il n'est pas rare de voir traiter des aliments d'origine végétaleet animale en milieu alcalin et à température élevée. Une telle exposition à la chaleur et à pH élevé est utilisée pour récupérer les protéines de céréales et de légumineuses, pour induire la formation de fibres, de protéines végétales analogues de viande, pour la préparation de fruits et légumes épluchés et pour la destruction des micro-organismes. En plus de leurs effets profonds sur les propriétés fonctionnelles et nutritionnelles dans les aliments, ces traitements peuvent aussi provoquer d'autres réactions secondaires, y compris la destruction des composés polyphénoliques naturels. Vu que les plantes contiennent un grand nombre de composés polyphénoliques, antioxydants, structurellement différents, anticancéreux et antimicrobiens, il serait intéressant de savoir si ces composés sont stables à la chaleur et à pH élevé (Mendel and Hella, 2000). En effet, les polyphénols sont des composés très réactifs (Zazo et al., 2005 ; Emilie, 2013). Les réactions qui démarrent dès la rupture de l'intégrité cellulaire des organes végétaux se poursuivent tout le long de leur conservation conduisant à une grande diversité de produits ajoutant à la complexité de la composition phénolique des végétaux. Les nouveaux composés formés présentent des propriétés pharmaceutiques et organoleptiques spécifiques, souvent distinctes de celles de leurs précurseurs. C'est pourquoi la connaissance de leur structure et des mécanismes par lesquels ils se forment est une base indispensable pour la maîtrise de leur utilisation aussi bien en agroalimentaire qu'en pharmacologie.

Les travaux de Mazza et Brouillard (1987) ont montré que l'acidité du milieu pouvait induire des changements structuraux. Par exemple, les anthocyanes présentent une couleur rouge en milieu acide et bleu en milieu neutre ou alcalin. L'utilisation industrielle de ces métabolites secondaires doit donc tenir compte de leur réactivité vis-à-vis de l'acidité du milieu et d'autres facteurs comme la lumière, la température et leur tendance à s'associer à de nombreuses autres molécules comme les protéines et les métaux. Plusieurs composé phénoliques appartenant à diverses familles ont été identifiés dans les différents organes du sorgho rouge (Waniska, 2000 ; Awika et Rooney, 2004 ; Dykes et Rooney, 2007 ; Bröhan et al., 2011 ; Pascal et al., 2012a). Mais malheureusement, ces molécules n'ont fait, à notre connaissance, l'objet d'aucune étude du point de vue stabilité. Cependant, l'extrait brut de la gaine foliaire du sorgho rouge est couramment utilisé dans diverses régions du Bénin et des pays voisins comme le Nigéria, le Togo, le Niger, etc, pour colorer le fromage laitier peuhl ou la bouillie de maïs. A cet effet, l'extraction est généralement faite en milieu alcalin et à forte température. La présente étude a donc été initiée pour évaluer la stabilité des composés phénoliques de l'extrait aqueux de la gaine foliaire du sorgho dans divers conditions de $\mathrm{pH}$ et de température.

\section{Matériel et method:-}

Le matériel utilisé dans cette étude est essentiellement constitué d'extrait phénolique de la gaine foliaire du sorgho et d'un spectrophotomètre UV-Visible à balayage de longueur piloté sur ordinateur par le logicielVarian scan. L'extraction a été réalisée en milieu aqueux qui est le meilleur milieu retenu lors de la phase d'optimisation de l'extraction dans une étude antérieure (Pascal et al., 2012b). L'extrait est ensuite dilué jusqu'à une concentration de $0,36 \mathrm{mg} / \mathrm{L}$ sur base de matière sèche afin de rester dans la limite de détection du spectrophotomètre. Trois échantillons ont été préparés à différents $\mathrm{pH}$ à partir de cette solution:

1. Echantillon $1:$ Solution conservée à $\mathrm{pH}$ neutre.

2. Echantillon 1 : Solution acidifiée par l'acide chlorhydrique et conservée à $\mathrm{pH}$ acide $(\mathrm{pH}=1)$.

3. Echantillon 1 : Solution alcalinisée par de l'hydroxyde de potassium conservée à $\mathrm{pH}$ basique $(\mathrm{pH}=11)$.

Pour étudier l'influence de la lumière et de la température sur les composés phénoliques au cours de la conservation, chacun des échantillons précédents est divisé en 3 parties et conservé dans différentes conditions de température et de luminosité:

1. une solution placée à basse température (au réfrigérateur à $4^{\circ} \mathrm{C}$ ),

2. une solution placée à l'obscurité à la température du laboratoire,

3. une solution placée à la lumière et à la température du laboratoire.

Soit au total 9 échantillons.

A raison d'une mesure par semaine, l'absorbance de chacune de ces solutions a été enregistrée au spectrophotomètre entre 250 et $750 \mathrm{mn}$ pendant 4 semaines. La comparaison des résultats de ces mesures nous permettra de déterminer la stabilité des extraits dans le temps et d'en déduire les conditions optimales de $\mathrm{pH}$, de température et de luminosité pour une meilleure conservation des différentes familles de composés phénoliques contenues dans ces extraits. 


\section{Résultats et discussion:-}

Aspect physique des extraits à différents pH

La couleur des extraits varie en fonction de la nature du milieu. Les solutions sont de couleur rose pâle en milieu neutre, orange en milieu acide et jaune milieu basique. Ces couleurs observées confirment l'existence des molécules de3-déoxyanthocyanes dans le sorgho comme signalé dans la littérature. Selon certains auteurs, ces changements de couleur sont dus aux équilibres chimiques qui s'établissent entre les différentes formes anthocyanidines dépendantes du pH : le cation flavylium, la base quinoïdale, la pseudobase ou carbinol et la chalcone (MAZZA, 1990 ; Jackman, 1996). Le passage d'une forme à l'autre fait intervenir des équilibres chimiques réversibles. En milieu fortement acide $(\mathrm{pH}<2)$ le cation flavylium est la seule espèce présente en solution. L'augmentation progressive du $\mathrm{pH}$ entraîne la coexistence de plusieurs formes et la couleur de la solution d'anthocyanes diminue en intensité. La concentration de chaque forme colorée ou incolores dépend des constantes d'équilibre qui contrôlent ces réactions d'équilibre acido-basiques : transfert de protons, hydratation et tautomérie (Choi, 2006). Ainsi, à pH élevé, les bases quinoniques se forment par perte d'un proton des hydroxyles libres en position 5,7 ou 4' de la forme colorée. Les bases quinoniques sont thermodynamiquement instables et se transforment en hémiacétals et en chalcones, en passant à nouveau par la forme du cation flavylium. La présence des molécules de 3-déoxyanthocyanes dans l'extrait du sorgho laisse prédire une relative stabilité de cet extrait face aux paramètres extérieurs tels que la température et le $\mathrm{pH}$.

\section{Comportement des extraits au cours des deux premières semaines de conservation}

Au jour $\mathrm{J}+0$ (jour de préparation des extraits), les spectres UV-Visible des échantillons aux trois $\mathrm{pH}$ ne présentent pas le même profil (Figure1). Cela met en évidence le fait que les composés phénoliques, en milieu acide, basique ou neutre, se présentent sous différentes formes en solution et absorbent à différentes longueurs d'onde.

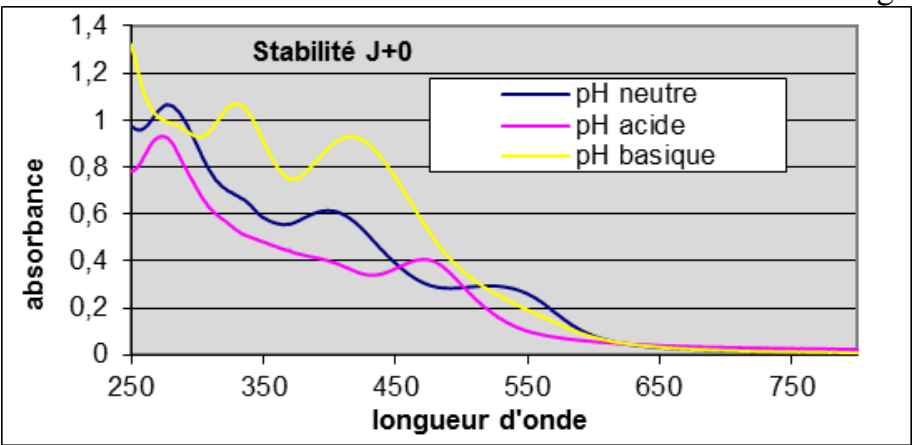

Figure1:-Spectres UV-visible de l'extrait de gaine foliaire à différents $\mathrm{pH}$

Quel que soit le $\mathrm{pH}$ considéré, on retrouve une bande d'absorption autour de $280 \mathrm{~nm}$ plus prononcée à $\mathrm{pH}$ neutre. Par contre, dans le Visible, les trois spectres montrent des différences significatives.

A pH neutre, on observe une bande de faible intensité à $330 \mathrm{~nm}$ correspondant aux phénylpropanoïdes et dérivés acylés, deux bandes d'intensité relativement moyenne à $405 \mathrm{~nm}$ et à $530 \mathrm{~nm}$ correspondant respectivement aux flavones puis aux anthocyanes et à leurs dérivés.

A pH basique, on observe une disparition de la bande à $530 \mathrm{~nm}$ contre une forte augmentation des absorbances à 330 $\mathrm{nm}$ et à $405 \mathrm{~nm}$. Ceci pourrait correspondre à une dégradation des anthocyanes en milieu fortement alcalin, conduisant à des dérivés acyles, des phénylpropanoïdes, des aglycones voir des chalcones absorbant à ces longueurs d'onde.

En milieu acide, on note une disparition quasi-totale de la bande d'absorption à $330 \mathrm{~nm}$, une baisse d'intensité de celle à $405 \mathrm{~nm}$ et un déplacement de celle à $530 \mathrm{~nm}$ vers les plus faibles longueurs d'onde $(470 \mathrm{~nm})$. Ceci serait essentiellement dû aux réactions d'hydrolyse en milieu acide.

L'analyse UV des 9 échantillons après une semaine de conservation ne montre aucune modification significative au niveau des bandes d'absorption, mis à part une légère diminution de leur intensité. Nous pouvons donc conclure que toutes les familles de composés initialement citées sont présentes, mais connaissent un début de dégradation qui se traduit par une légère baisse d'intensité des absorptions. Les mesures réalisées après la deuxième semaine de 
conservation montrent des résultats analogues à ceux de la première semaine, avec une dégradation un peu plus poussée.

\section{Comportement des extraits au cours de la troisième semaine de conservation}

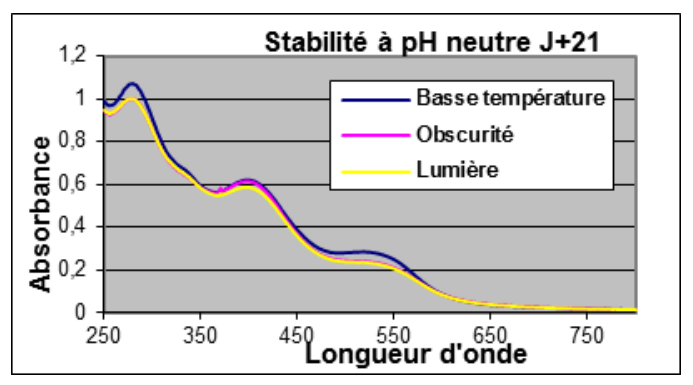

a-

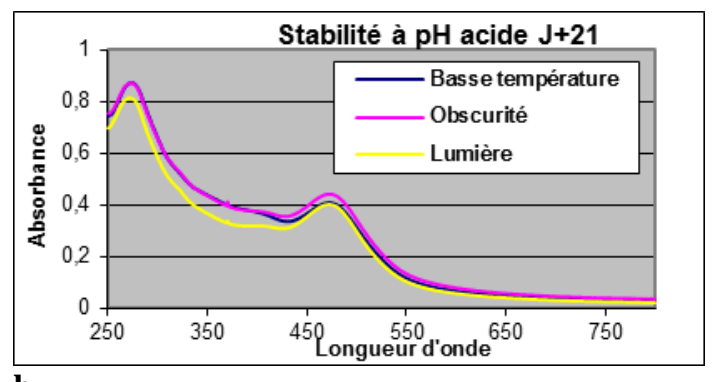

b-

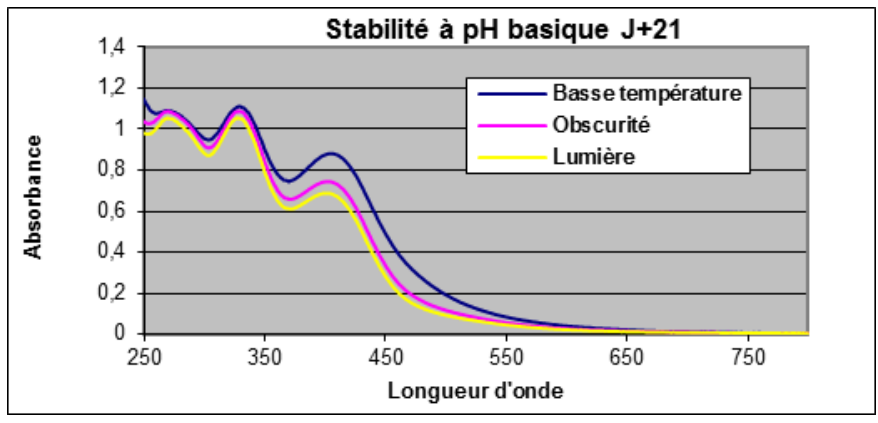

c-

Figure2 :-Spectres UV-Visible de l'extrait à $\mathrm{pH}$ neutre (a), acide (b) et basique (c) après 21 jours de conservation dans différentes conditions de température et de lumière.

La différence entre les spectres, très faible les deux premières semaines, commence à se faire de plus en plus remarquer à la troisième semaine.

A pH neutre, On note que la solution gardée à basse température commence à avoir des absorbances plus élevées que les deux autres qui ne diffèrent pratiquement pas entre elles. Cela pourrait signifier qu'à ce $\mathrm{pH}$ les produits se conservent mieux à basse température qu'à température ambianteet que la lumière a moins d'incidence. Par contre, à $\mathrm{pH}$ acide, on constate un effet significatif de la lumière. La solution placée à la lumière et à température ambiante semble se dégrader plus vite que les deux autres qui restent quasiment aux mêmes absorbances. Les composés phénoliques contenus dans l'extrait montrent donc une sensibilité plus forte à la lumière qu'à la température à $\mathrm{pH}$ acide. A pH basique, on observe encore plus de différence ; les trois courbes se détachent nettement, surtout dans le domaine du visible. La solution laissée à basse température est bien la plus stable suivie de celle conservée à l'obscurité. Nous pouvons donc conclure qu'en milieu basique, tout comme en milieu neutre, et par opposition au milieu acide, la température a un effet plus marqué que la lumière sur la conservation des composés phénoliques contenus dans l'extrait. 


\section{Comportement des extraits après quatre semaines de conservation}

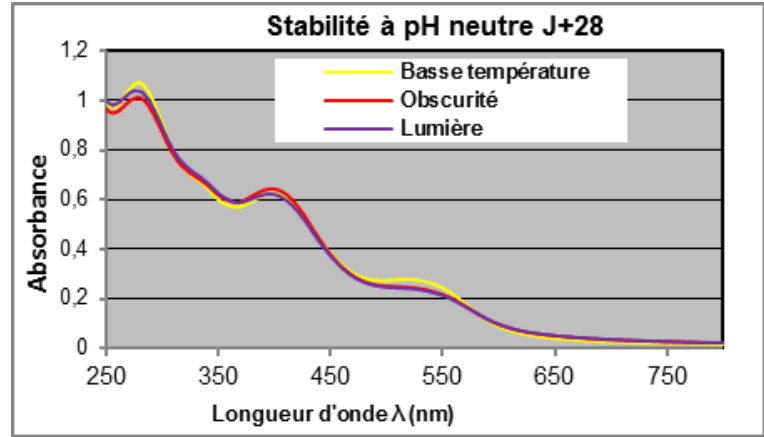

a-

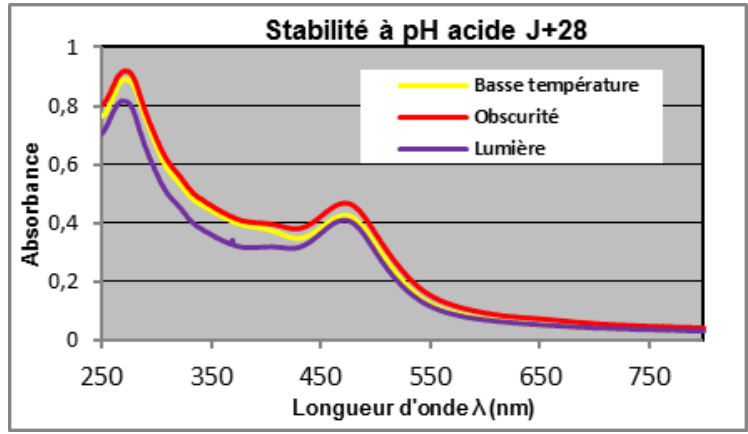

b-

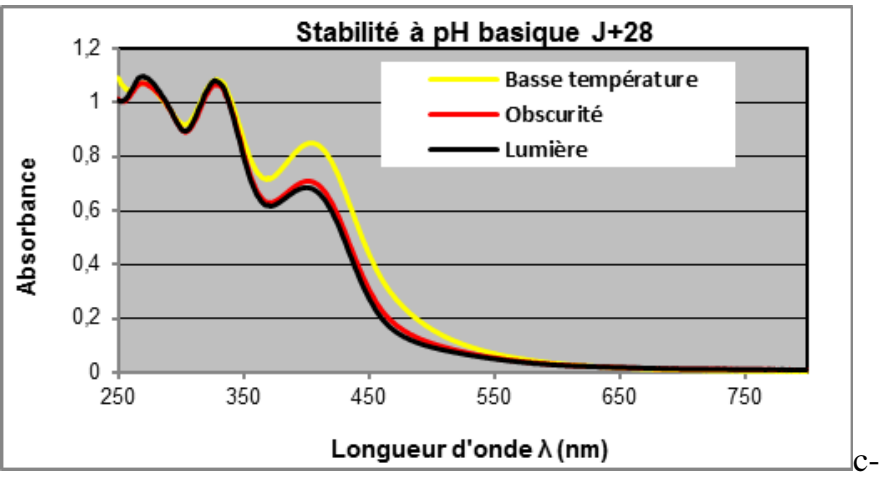

Figure3 :-Spectres UV-Visible de l'extrait à $\mathrm{pH}$ neutre (a), acide (b) et basique (c) après 28 jours de conservation dans différentes conditions de température et de lumière.

A pH neutre, les flavones semblent n'avoir aucune sensibilité à la température de conservation et une faible sensibilité à la lumière. Par contre les anthocyanes bien que quasiment insensibles à la lumière, subissent une dégradation plus ou moins importante lorsqu'elles sont conservées à température ambiante pendant 28 jours.La température semble avoir moins d'effet que la lumière sur la conservation des anthocyanes du sorgho en milieu acide.

Les observations faites après deux semaines de conservation s'accentuent au bout de quatre semaines. La lumière influence très peu la conservation des composés phénoliques contenus dans l'extrait en l'occurrence celle des flavones et des polyphénols totaux.

Afin d'évaluer la stabilité des composés phénoliques contenus dans les extraits en fonction du temps, nous avons tracé pour chaque famille de composés phénoliques le pourcentage de composés restant en fonction du temps dans les différentes conditions de conservation. 


\section{Cinétique de décomposition des anthocyanes à différents pH}
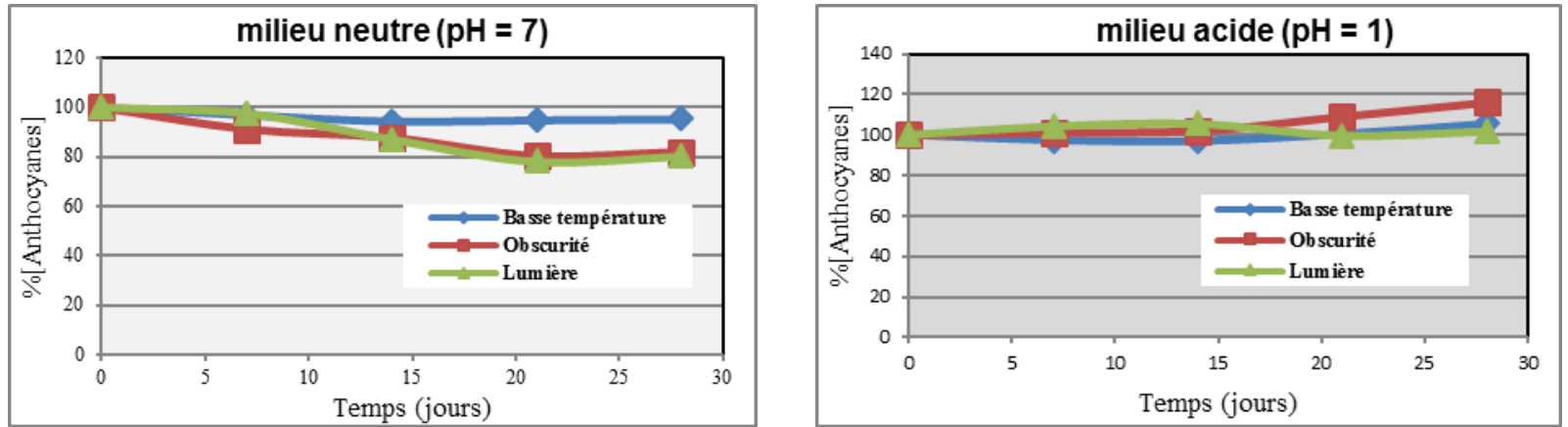

Figure4 :-Cinétique de dégradation des anthocyanes en milieu neutre et en milieu acide

On constate qu'à $\mathrm{pH}$ neutre, les anthocyanes se conservent mieux à froid (moins de 5\% de dégradation) qu'à température ambiante (18 à 20\% de dégradation). Mais à température égale, la lumière n'a pratiquement pas d'effet sur les pigments anthocyaniques du sorgho (moins de $2 \%$ de dégradation à la lumière après 28 jours de conservation). Par contre, à $\mathrm{pH}$ acide, la lumière à une influence plus marquée que la température après deux semaines de conservation. Par ailleurs, nous avons remarqué que quelles que soient les conditions de température et de lumière testées, les anthocyanes se conservent mieux à $\mathrm{pH}$ acide qu'à $\mathrm{pH}$ neutre. Cette observation confirme les résultats d'autres chercheurs qui ont démontré que les anthocyanes sont plus stables à $\mathrm{pH}$ acide qu'à $\mathrm{pH}$ basique. Cette relative stabilité des anthocyanes du sorgho serait due à leur forme combinée sous forme d'hétéroside, on parle alors d'anthocyanines. Selon certains auteurs, les glycosylations rencontrées chez les anthocyanines leur confèrent leur solubilité et leur stabilité (Markakis, 1982 ; Cevallos-Casals et Cisneros-Zevallos, 2004).

\section{Cinétique de décomposition des flavones à différents pH}

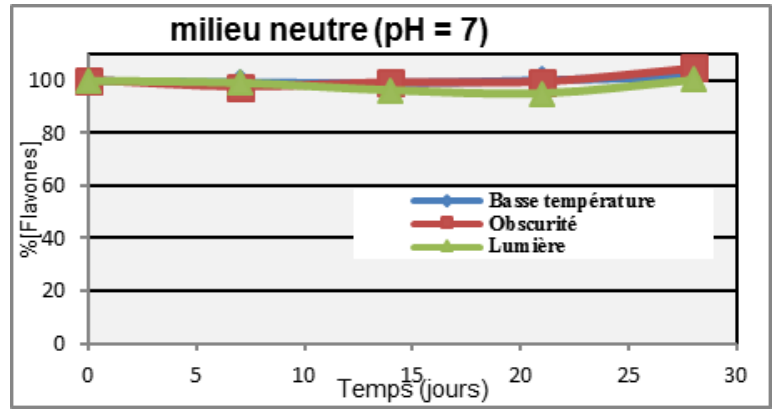

a-

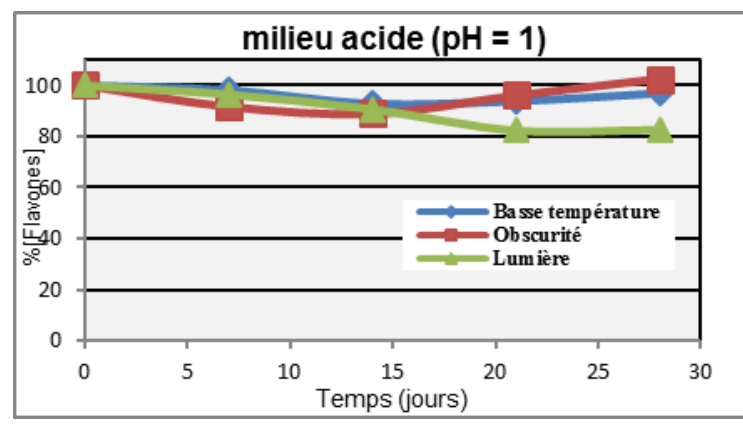

b-

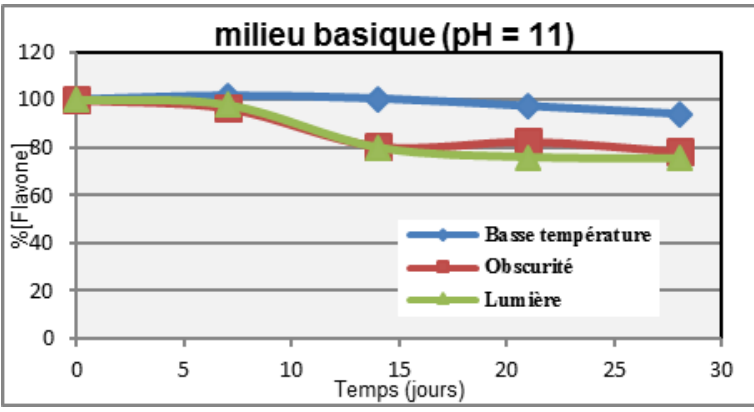

c-

Figure5 :-Cinétique de dégradation des Flavones en milieu neutre (a), acide (b)et basique (c) 
Contrairement aux anthocyanes, les flavones montrent une sensibilité plus marquée à la lumière qu'à la température et se conservent mieux en milieu neutre qu'en milieu acide. A pH neutre, aucune dégradation n'est observée après 28 jours et à température ambiante.Une décomposition de moins de $5 \%$ est observée à la lumière à ce $\mathrm{pH}$. Mais à $\mathrm{pH}$ acide, $3 \%$ se décomposent à basse température et $18 \%$ à la lumière et à température ambiante. Le milieu basique est le moins indiqué pour la conservation des flavones avec 5\% de décomposition à basse température et 20 à $25 \%$ de décomposition provoquée par la lumière et la température ambiante.

\section{Cinétique de décomposition des composéspolyphénols totauxà différents pH}

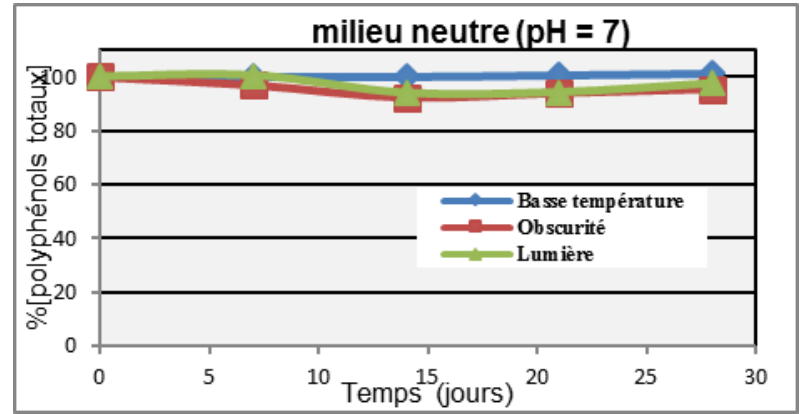

a-

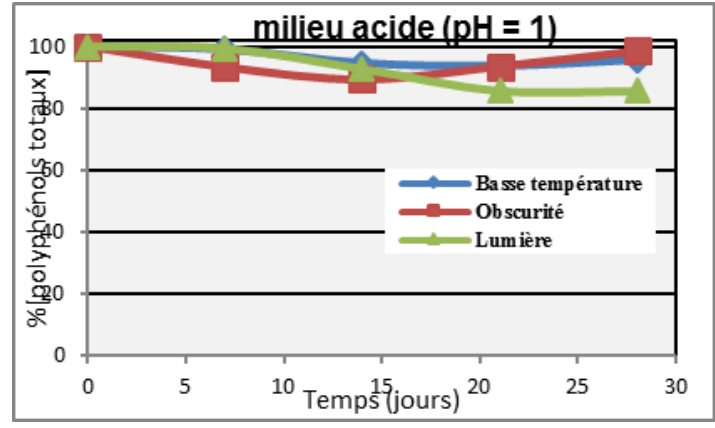

b-

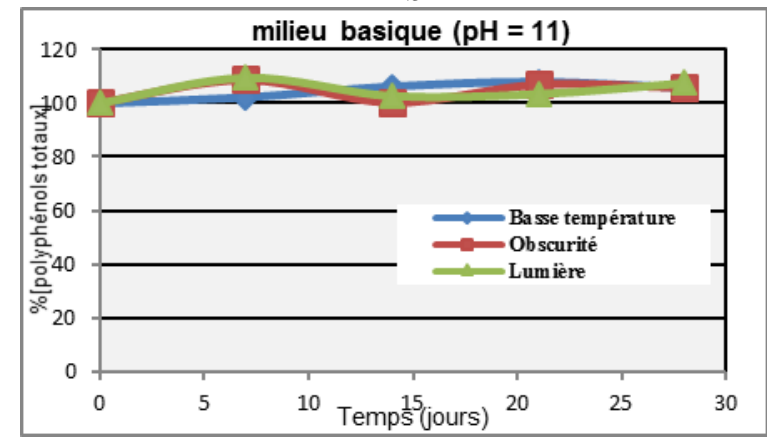

c-

Figure 2 :-Cinétique de dégradation des polyphénols totauxen milieu neutre (a), acide (b) et basique (c)

En milieu neutre, on n'observe pratiquement pas de modification de la concentration des polyphénols totaux après 28 jours de conservation à basse température. Seulement 3 à $5 \%$ de décomposition ont été induits par la lumière et la température ambiante. Mais à $\mathrm{pH}$ acide on note une baisse allant de 2 à $15 \%$ de la teneur en polyphénols totaux causée par la lumière et la température. Une montée de la teneur en composés phénoliques dépassant les $100 \%$ a été constatée en milieu basique. Nous assimilons ce phénomène à la décomposition observée au niveau des grosses molécules de flavonoïdes, dont les anthocyanes, libérant plus de composés phénoliques de tailles moyennes, augmentant ainsi leur concentration et, par conséquent, celle des composés phénoliques totaux.

\section{Conclusion:-}

A l'issu de ce travail nous pouvons conclure que les composés phénoliques du sorgho se comportent différemment selon leur famille d'appartenance vis-à-vis des conditions de conservation. Les flavones, composés absorbant à 330 $\mathrm{nm}$, semblent assez peu influencés par les effets de la lumière et de la température. Leur stabilité ne semble pas très affectée par ces paramètres.Les anthocyanes essentiellement de nature 3-déoxy (absorption à $530 \mathrm{~nm}$ en milieu neutre et à $470 \mathrm{~nm}$ en milieu acide) sont plus sensibles à la température qu'à la lumière à pH neutre et présentent un comportement inverse en milieu acide.

Quel que soit le milieu, la conservation à basse température (au réfrigérateur à $4{ }^{\circ} \mathrm{C}$ ) est conseillée. Toutefois, il faut signaler que les polyphénols du sorgho sont des composés relativement stables vis-à-vis de la lumière avec moins de $20 \%$ de décomposition après 28 jours de conservation à température ambiante, ceci indépendamment des conditions de $\mathrm{pH}$. 


\section{Références:-}

1. Céline Chanforan. (2011). Stabilité de microconstituants de la tomate (composés phénoliques, caroténoïdes, vitamines $\mathrm{C}$ et $\mathrm{E}$ ) au cours des procédés de transformation: études en systèmes modèles, mise au point d'un modèle stoechio-cinétique et validation pour l'étape unitaire de préparation de sauce tomate. Thèse de doctorat, Université d'Avignon, France 400p.

2. Pascal C. D. AGBANGNAN, Silvia ALEXANDROVA, Anna CHROSTOWSKA, Christine TACHON, Eric FOUQUET, Dominique C. K. SOHOUNHLOUE (2011). Concentration des polyphénols du sorgho par filtration membranaire : cas de l'ultrafiltration ; Récents Progrès en Génie des Procédés, 101, ISSN 1775-335 - ISBN 2910239-75-6, Ed. SFGP, Paris, France.

3. Singleton V. L., Ortofer R., Lamuela-Raventos R. M.; (1999). Analysis of total phenols and other oxidation substrates and antioxidants by means of Folin-Ciocalteu reagent. Packer L (ed) Methods in enzymology. Orlando Academic Press London: 152-78.

4. Kabran G.R., Ambeu N. C., Mamyrbékova-Békro J. A., Békro Y. A. (2011). CCM des extraits sélectifs de 10 plantes utilisées dans le traitement traditionnel du cancer du sein en Côte d'Ivoire. European Journal of Scientific Research 63(4): 592-603.

5. Fahima Ali-R ACHEDI, Souad MERAGHNI, Nourhène TOUAIBIA et Sabrina M. (2018). Analyse quantitative des composés phénoliques d'une endémique algérienne ScabiosaAtropurpureasub. Maritima L. Bulletin de la Société Royale des Sciences de Liège, 87, 13 -21

6. Mendel Friedman and Hella S. Jürgens. (2000). Effect of pH on the Stability of Plant Phenolic Compounds. Journal of Agricultural and Food Chemistry. 48, $2101-2110$.

7. J. A. Zazo, J. A. Casas, A. F. Mohedano, M. A. Gilarranz, and J. J. Rodríguez (2005). Chemical Pathway and Kinetics of Phenol Oxidation by Fenton's Reagent, Environmental Science \& Technology, 39(23).

8. Emilie Petit (2013). Réactivité de polyphénols du vin sous conditions oxydantes : hémisynthèse des mongolicaïnes, et d'adduits entre polyphénols et thiols odorants. Thèse de doctorat, Institut des Sciences Moléculaires, Université Sciences et Technologies - Bordeaux I, France.

9. G. Mazza, and Raymond Brouillard; (1987). Color stability and structural transformations of cyanidin 3,5diglucoside and four 3-deoxyanthocyanins in aqueous solutions. Journal of Agricultural and Food Chemistry, $35(3), 422-426$.

10. Waniska R.D., (2000). Technical and institutional options for sorghum grain mold management. In: Chandrashekar A., Bandyopadhyay R. \& Hall A.J., eds. Proceeding of an international consultation, 18-19 May 2000, ICRISAT, Patancheru, India. Patancheru, India: ICRISAT, 72-106.

11. Awika J.M. \& Rooney L.W., (2004). Sorghum phytochemicals and their potential impact on human health. Phytochemistry, 65, 1199-1221.

12. Dykes L. \& Rooney L.W., (2007). Phenolic compounds in cereal grains and their health benefits. Cereal Foods World, 52(3), 105-111.

13. Bröhan M., Jerkovic V. \& Collin S., (2011). Potentiality of red sorghum for producing stilbenoid-enriched beers with high antioxidant activity. J. Agric. Food Chem., 59, 4088-4094.

14. Pascal D. C. Agbangnan, Christine Tachon, Hélène Bonin, Anna Chrostowska, Eric Fouquet, Dominique C. K. Sohounhloue (2012b). Phytochemical study of a tinctorial plant of Benin traditional pharmacopoeia: The red sorghum (sorghum caudatum) of Benin; Scientific Study \& Research; 13(2), 121-135.

15. Pascal C. Agbangnan D., Christine Tachon, Justine Dangou, Anna Chrostowska, Eric Fouquet, Dominique C. K. Sohounhloue (2012b). Optimization of the extraction of sorghum's polyphenols for industrial production by membrane processes; Research Journal of Recent Sciences; 1(4), 1-8.

16. MAZZA G., BROUILLARD R., (1990). The mechanism of co-pigmentation of anthocyanins in aqueous solutions.Phytochemistry; 29, 1097-1102.

17. Jackman, R. L.; Smith, J. L. Anthocyanins and Betalains. InNatural Food ColorantsHendry, G. A. F., Houghton, J. D., Eds.; Blackie Academic and Professional: Glasgow, U.K., 1996; pp 244-280.

18. Choi, J. S. (2006). Antioxidant activity of methanolic extracts from some grains consumed in Korea. Phytochemistry; 103, 130-138.

19. Markakis P. "Stability of anthocyanins in foods," (1982).Anthocyanins as food colours New York, Academic Press, 245-253.

20. Cevallos-Casals B. A., Cisneros-Zevallos L (2004). Extraction and Stability of Anthocyanins Present in the Skin of the Dragon Fruit (Hylocereusundatus).Food Chemistry, 86, 69-77. 